\title{
Neonatal cardiac dysfunction in intrauterine growth restriction
}

\author{
Sotirios Fouzas ${ }^{1,2}$, Ageliki A. Karatza ${ }^{1,2,3}$, Periklis A. Davlouros ${ }^{4}$, Dionisios Chrysis², Dimitrios Alexopoulos ${ }^{4}$, Stefanos Mantagos ${ }^{1,2}$ \\ and Gabriel Dimitriou ${ }^{1,2}$
}

BACKGROUND: The early postnatal cardiovascular consequences of intrauterine growth restriction (IUGR) have not been completely elucidated. This study aimed to evaluate the effect of IUGR on neonatal myocardial function and cardiovascular adaptation to extrauterine life.

METHODS: Conventional and tissue Doppler echocardiographic parameters were compared on the second and fifth postnatal day between 30 IUGR and 30 appropriate-forgestational age (AGA) neonates.

RESULTS: IUGR neonates presented relative interventricular septum (IVS) hypertrophy (IVS to left ventricular (LV) posterior wall diastolic ratio: median IUGR-AGA difference of 0.05 (interquartile range: $0.04-0.06) ; P=0.020$ ), relative $\mathrm{LV}$ dilatation (wall thickness to end-diastolic LV dimension difference of 0.12 (0.06$0.16) ; P=0.012)$, and increased left myocardial performance index (MPI difference of $0.19(0.05-0.28) ; P=0.012)$. Repeated measurements ANOVA revealed a different pattern of change in LV stroke volume (LVSV; $P<0.001$ ), LV cardiac output (LVCO; $P<$ 0.001), MPI $(P<0.001)$, and heart rate $(H R ; P=0.025)$ between $A G A$ and IUGR infants. From the second to the fifth postnatal day, AGA neonates presented a decrease in MPI and HR with an increase in LVSV and LVCO. IUGR neonates failed to achieve similar changes in MPI, HR, and LVSV, whereas their LVCO decreased. CONCLUSION: IUGR neonates present changes in cardiac morphology and subclinical myocardial dysfunction, which may result in an altered pattern of cardiovascular adaptation to extrauterine life.

ntrauterine growth restriction (IUGR), defined as the inability of a fetus to achieve its genetically determined potential size, is associated with increased risk of perinatal complications and neonatal morbidity (1). Epidemiological evidence also suggests a strong relationship between IUGR and cardiovascular disease in adulthood (2), supporting the existence of a maladaptive programming process in utero, which affects cardiovascular system in the long term (3). Previous studies have shown that chronic intrauterine substrate deprivation is associated with changes in ventricular geometry and "cardiomyopathy-like" myocardial dysfunction in the fetus (4-7). Alterations in cardiovascular adaptation to extrauterine life have also been reported in neonates exposed to IUGR, but these findings were only hypothetically related to an impairment in myocardial function (8).

Traditionally, the assessment of cardiac performance in infants relies on conventional echocardiographic indexes (9). Tissue Doppler imaging (TDI) is a relatively new echocardiographic technique, which enables assessment of ventricular mechanics by providing information on myocardial motion and timing of cardiac events with high temporal and spatial resolution (10). Accumulating evidence from studies in neonates (11-15) and children (16-18) suggests that specific TDI parameters, such as peak myocardial velocities and myocardial performance index (MPI) (19), are more sensitive markers of ventricular dysfunction than those derived from conventional echocardiography.

In this study, using both conventional and TDI echocardiography, we sought to evaluate cardiac morphology and myocardial performance in neonates with and without IUGR and to compare their pattern of cardiovascular adaptation after birth. We hypothesized that IUGR neonates present structural and functional cardiac changes, which might be more prominent during the high-demand transitional period and may influence cardiovascular adaptation to extrauterine life.

\section{RESULTS}

\section{Baseline Characteristics}

Participants' baseline characteristics and between-group comparisons are presented in Table 1. Tobacco smoke exposure during pregnancy, abnormal umbilical artery Doppler, and delivery by cesarean section were more frequently noted in the IUGR group.

\section{Outcomes on the Second Postnatal Day}

The IUGR neonates had higher IVSd/LVPWd ratio (relative interventricular septum (IVS) hypertrophy) and lower relative wall thickness (relative left ventricular (LV) dilatation) compared 


\section{Articles $\mid$ Fouzasetal.}

Table 1. Baseline characteristics of the study groups

\begin{tabular}{|c|c|c|c|}
\hline & $\operatorname{AGA}(n=30)$ & $\operatorname{IUGR}(n=30)$ & $P$ value \\
\hline \multicolumn{4}{|l|}{ Maternal and pregnancy characteristics } \\
\hline Maternal age (y) & $32(29-34)$ & $31(28-34)$ & 0.711 \\
\hline Smoking during pregnancy (\%) & 10.0 & 33.3 & 0.028 \\
\hline Gestational diabetes (\%) & 3.3 & 10.0 & 0.612 \\
\hline Absent or reversed end-diastolic flow in umbilical artery Doppler (\%) & - & 20.0 & - \\
\hline Prenatal glucocorticoid administration (\%) & 30.0 & 53.3 & 0.067 \\
\hline \multicolumn{4}{|l|}{ Neonatal characteristics } \\
\hline Male sex (\%) & 46.7 & 40.0 & 0.602 \\
\hline Gestational age at delivery, week (range) $^{b}$ & $36(32-39)$ & $36(32-39)$ & - \\
\hline Birth weight (g) & $2,805(2,170-3,240)$ & $1,640(1,240-1,970)$ & $<0.001$ \\
\hline Birth length $(\mathrm{cm})$ & $48.5(45-50)$ & $43.5(39-45)$ & $<0.001$ \\
\hline Ponderal index $\left(\mathrm{kg} / \mathrm{m}^{3}\right)$ & $25.2(23.0-27.3)$ & $21.1(19.3-23.1)$ & $<0.001$ \\
\hline Apgar score at $1 \mathrm{~min}$ & $9(8-9)$ & $9(8-9)$ & 0.895 \\
\hline Apgar score at $5 \mathrm{~min}$ & $10(9-10)$ & $10(9-10)$ & 0.534 \\
\hline Hemoglobin $(\mathrm{g} / \mathrm{dl})^{c}$ & $15.8(15.0-17.2)$ & $16.7(15.0-17.6)$ & 0.355 \\
\hline \multicolumn{4}{|l|}{ Minor neonatal morbidities } \\
\hline Oxygen requirement (\%) ${ }^{d}$ & 26.7 & 20.0 & 0.542 \\
\hline PDA (\%) & 6.7 & 10 & 0.999 \\
\hline
\end{tabular}

Values are median (interquartile range) unless stated otherwise.

AGA, appropriate-for-gestational age; IUGR, intrauterine growth restriction; PDA, patent ductus arteriosus.

aCalculated by $x^{2}$, Fisher's exact, or Mann-Whitney U-test, as appropriate. 'bUGR-control pairs were matched for gestational age. ${ }^{\circ}$ Measured at 24 postnatal hours. ${ }^{\mathrm{d} D}$ Duration of oxygen requirement less than $12 \mathrm{~h}$. ${ }^{\mathrm{e}}$ Only for infants with PDA.

with appropriate-for-gestational age (AGA; Table 2). No differences were noted in LV ejection fraction and fractional shortening between the two groups. Higher LV stroke volume (LVSV), higher heart rates, and higher LV cardiac output (LVCO) were documented in the IUGR group. The IUGR neonates had reduced $S^{\prime}$ and $\mathrm{E}^{\prime}$ myocardial velocities, higher $\mathrm{E} / \mathrm{E}^{\prime}$ ratios, prolonged isovolumetric contraction time and isovolumetric relaxation time, and significantly higher MPI for both ventricles as compared with AGA (Table 2). Typical TDI findings from an AGA neonate and a neonate with IUGR are shown in Figure 1. The IUGR group presented significantly higher B-type natriuretic peptide (BNP) levels, which were positively related to LV MPI (linear regression coefficient: $0.83 ; R^{2}: 0.69 ; P<0.001$ ). Mean blood pressure was higher in the neonates with IUGR (Table 2).

\section{Outcomes on the Fifth Postnatal Day and Second-to-Fifth Day Comparisons}

The pattern of change in LVSV, LVCO, MPI, and heart rate from the second to the fifth postnatal day was different between AGA and IUGR infants (Figure 2). By the fifth postnatal day, AGA neonates presented a significant increase in LVSV and LVCO and a decrease in heart rate. On the contrary, LVSV remained stable in the IUGR group, whereas LVCO decreased. Consequently, on the fifth postnatal day, the LVCO was comparable between the two groups although the heart rate remained significantly higher in the IUGR group (Table 2). Similarly to the second postnatal day, the IUGR neonates presented reduced myocardial velocities, higher $\mathrm{E} / \mathrm{E}^{\prime}$ ratios, prolonged isovolumetric relaxation time and isovolumetric contraction time, and significantly higher MPI (Table 2). LV MPI decreased significantly in AGA neonates but remained unchanged in those with IUGR (Figure 2). BNP levels were again significantly higher in IUGR infants (Table 2) and positively correlated with LV MPI (regression coefficient: $0.66 ; R^{2}: 0.43 ; P<0.001$ ). By the fifth postnatal day, mean blood pressure was comparable between the two groups (Table 2), mainly due to the significant increase in AGA (Figure 2). 
Table 2. Cardiovascular outcomes on the second and fifth postnatal day

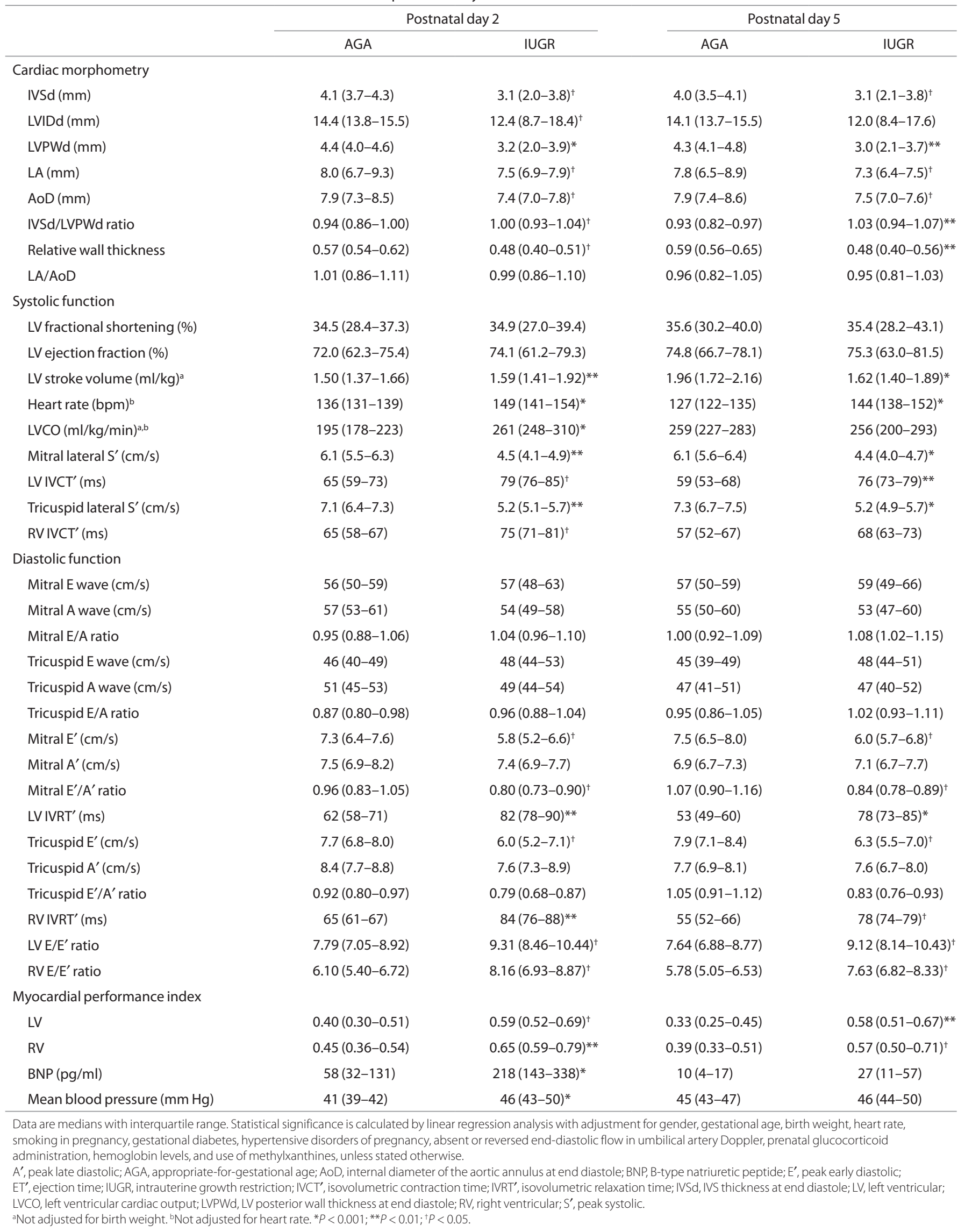



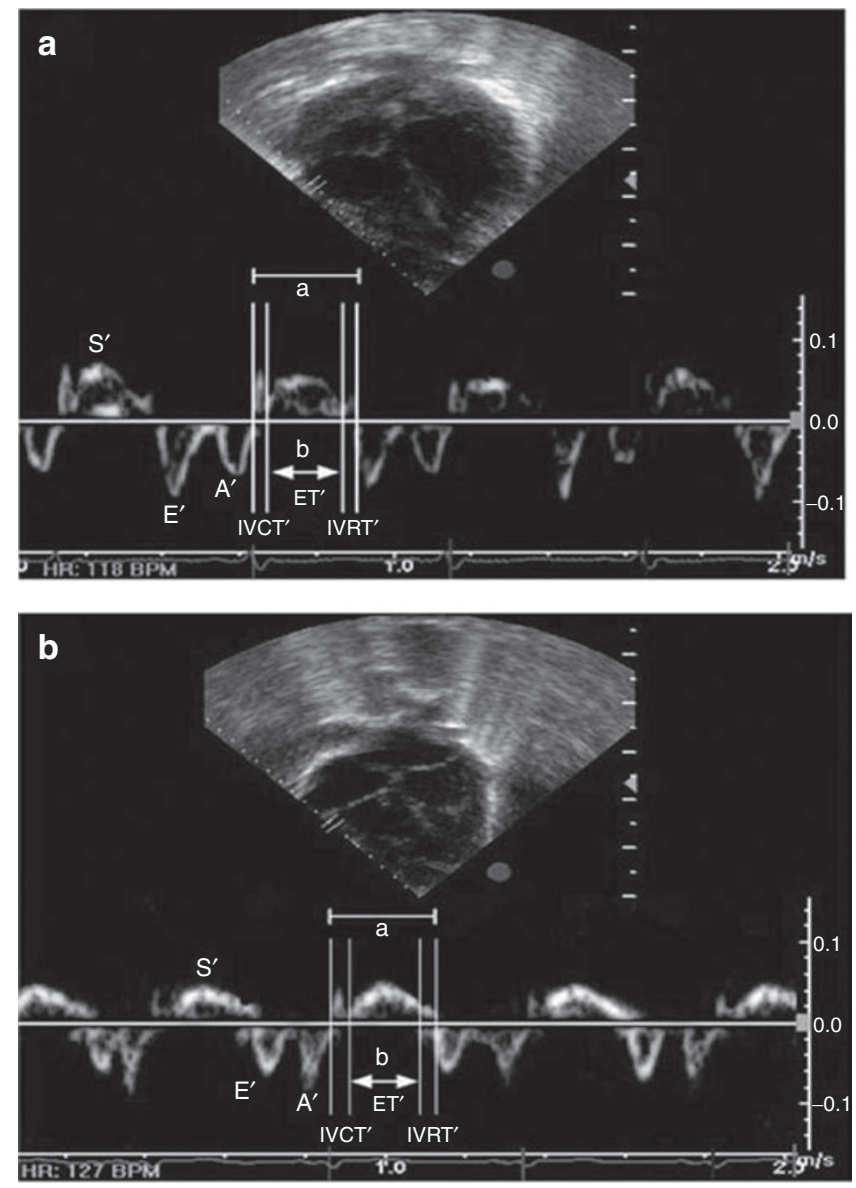

Figure 1. Tissue Doppler echocardiographic images (lateral tricuspid annulus) from (a) an appropriate-for-gestational age and (b) an intrauterine growth restriction neonate. Peak systolic $\left(S^{\prime}\right)$, peak early diastolic $\left(E^{\prime}\right)$, and peak late diastolic $\left(A^{\prime}\right)$ velocities are illustrated. Differences in isovolumetric contraction time (IVCT'), isovolumetric relaxation time (IVRT'), ejection time ( $\left.E T^{\prime}\right)$, and the amplitude of $E^{\prime}, A^{\prime}$, and $S^{\prime}$ waves between the two cases are noted. The interval $a$ is the time interval between the cessation of end-diastolic velocity and the onset of diastolic velocity $\left(a=\mathrm{IVCT}^{\prime}+\mathrm{ET}^{\prime}\right.$ + IVRT'). The interval $b$ represents the time interval between onset and cessation of systolic velocity $\left(b=\mathrm{ET}^{\prime}\right)$. Myocardial performance index was calculated as $(a-b) / b(19)$.

\section{DISCUSSION}

The IUGR neonates of our study presented distinct changes in cardiac morphology as reflected by the relative IVS hypertrophy and LV dilatation. Similar echocardiographic findings have previously been reported in neonates and children exposed to IUGR $(8,17,18)$, whereas chronic intrauterine substrate deprivation has been associated with alterations in cardiac geometry in human fetuses (7) and animal models (20). Exposure to increased myocardial workload in utero has been proposed to be the most probable pathophysiological mechanism for these changes $(7,8,20)$. Chronic intrauterine hypoxia results in increased placental vascular resistance (7), redistribution of cardiac output in favor of the left fetal heart (21), alterations of arterial structure and vascular tone regulation (22), and disturbances that contribute to the development of cardiac hypertrophy (23). The remodeling process may also be an adaptive response to reduced substrate supply
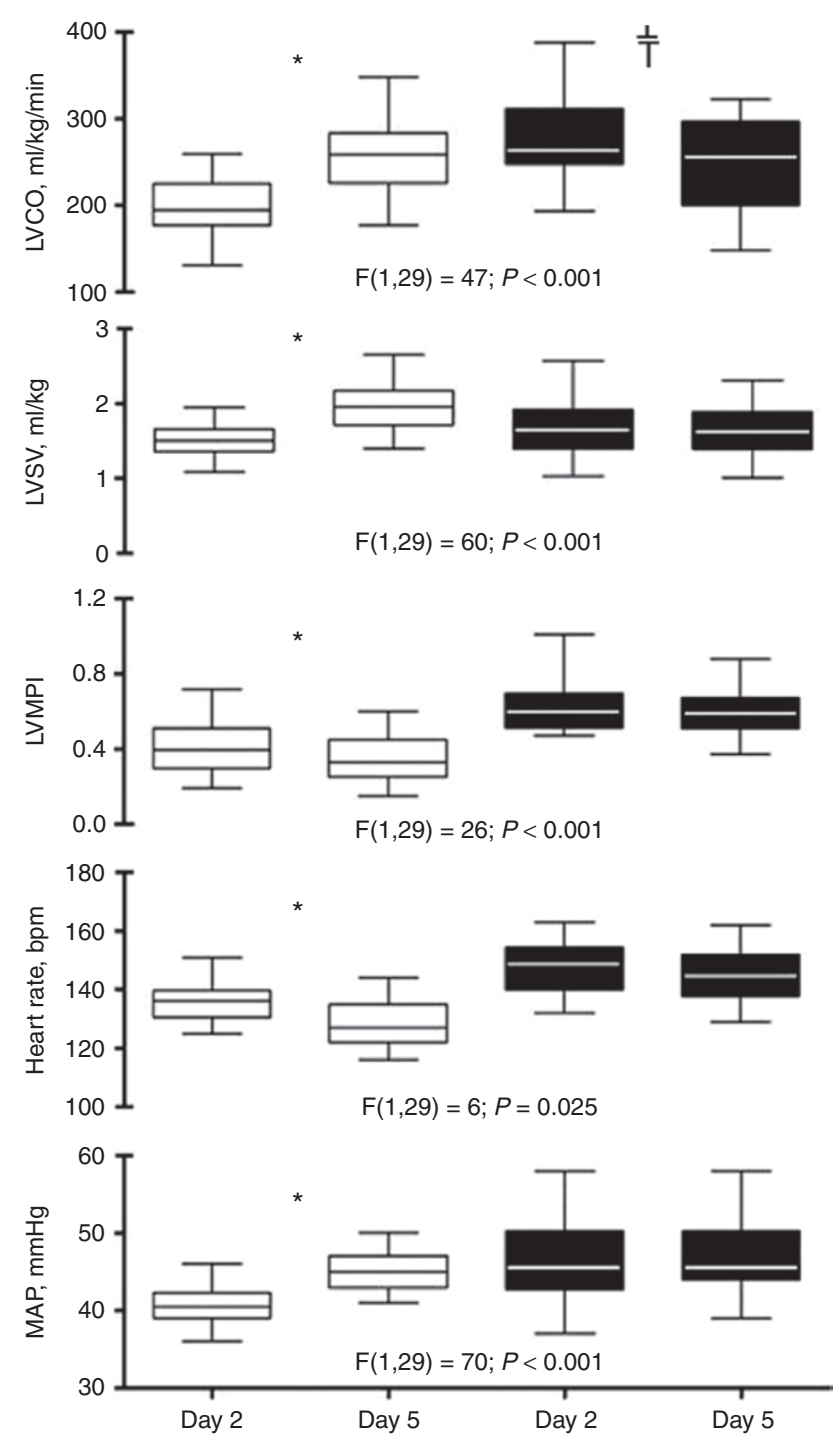

Figure 2. Changes in left ventricular cardiac output (LVCO), left ventricular stroke volume (LVSV), left ventricular myocardial performance index (LVMPI), heart rate, and mean arterial pressure (MAP) between second and fifth postnatal day in appropriate-for-gestational age (white boxes) and intrauterine growth restriction (black boxes) neonates. The results of repeated measurements ANOVA for assessing between-groups changes over time are presented. ${ }^{*} P<0.001 ;{ }^{\dagger} P<0.05$ for within-groups second-tofifth postnatal day comparisons (paired $t$-test).

through the modulation of specific gene expression at the myocardial level (24).

Recent research has strongly suggested that cardiac remodeling results in alterations of myocardial architecture and function $(20,23)$, which might explain the "cardiomyopathylike" findings reported in growth-restricted fetuses (4-6). Yet, direct echocardiographic evidence of cardiac dysfunction has not been reported to date in neonates exposed to IUGR (8). Although in our study, conventional echocardiographic parameters were not different between AGA and IUGR neonates, TDI echocardiography revealed that the IUGR neonates had impaired longitudinal axis function, remarkably abnormal myocardial relaxation, and decreased global cardiac performance as compared with AGA controls. These findings were 
consistently present on the second and fifth postnatal day, independently of factors such as prematurity, birth weight, and gestational or neonatal morbidities. To the best of our knowledge, this study is the first to utilize TDI echocardiography for assessing myocardial function in IUGR neonates.

Postnatal cardiovascular adaptation in the AGA neonates was characterized by a significant increase in LVSV with a concomitant decrease in heart rate. On the contrary, neonates exposed to IUGR failed to achieve a similar increase in LVSV and managed to preserve LVCO only by sustaining higher heart rates. Although similar patterns of altered hemodynamic adaptation have previously been reported for IUGR neonates $(8,25)$, in this study, these disturbances are clearly related to an underlying impairment in myocardial function. Indeed, the increase of LVSV in AGA neonates occurred in parallel with the improvement of global myocardial performance (i.e., decrease in MPI), while the failure of LVSV increase in their IUGR peers was associated with the lack of a similar augmentation. These findings suggest that neonates exposed to IUGR may have limited myocardial reserves at birth, which may influence cardiovascular adaptation during the high-demand transitional period and predispose to subsequent cardiovascular compromise.

The initially higher LVCO in IUGR neonates is most likely a consequence of the redistribution of cardiac output in favor of the left fetal heart to ensure adequate metabolic supply in vital organs (21). Transductal and interatrial shunts may also influence LVCO due to the resultant increase in ventricular preload. However, this is most likely not the case in our study because infants with a hemodynamically significant patent ductus arteriosus were excluded. Hemodynamically nonsignificant patent ductus arteriosus was still present in $6.7 \%$ of the AGA neonates and in $10 \%$ of the IUGR group, but with a maximum ductal diameter which in no case exceeded $0.8 \mathrm{~mm} / \mathrm{kg}$ body weight (Table 1). Moreover, the left atrial-to-aortic root diameter ratio, which represents a reliable echocardiographic index of left atrial volume overload, was comparable between AGA and IUGR infants (Table 2).

Serum BNP increases under conditions of hemodynamic stress associated with ventricular enlargement with or without cardiac dysfunction $(26,27)$. BNP levels also surge shortly after birth but decline rapidly on the first postnatal days, suggesting a crucial regulatory role of this molecule in the hemodynamic changes associated with transition to extrauterine life $(27,28)$. In our study, the IUGR neonates had significantly higher BNP levels on both second and fifth postnatal day as compared with AGA infants, a finding that is in agreement with a previous report (8). Moreover, BNP levels in infants exposed to IUGR were significantly correlated with LV MPI, supporting further the presence of a subtle myocardial dysfunction in this group.

Taken together, the findings of this study support the hypothesis that IUGR is associated with a maladaptive programming process with direct negative effects on the developing cardiovascular system $(2,3)$. The intrauterine effects of this process have been clearly established (4-6), but its immediate postnatal consequences have not been completely elucidated (8).
Our study provides evidence that neonates exposed to IUGR present distinct changes in cardiac morphology at birth andmost important-that these changes are associated with a subclinical myocardial dysfunction that affects cardiovascular adaptation to extrauterine life. Given that similar alterations in cardiac shape and function have been also described in older children exposed to IUGR $(17,18)$, the prognostic importance of our findings-especially in light of a potential interaction between the IUGR-related metabolic and cardiovascular programming in later life $(2,3)$-remains to be determined.

This study has some limitations. First, our case subjects consisted of neonates with proven IUGR but with minor neonatal morbidities. Therefore, firm conclusions regarding myocardial dysfunction and altered cardiovascular adaptation in more severe clinical forms of IUGR cannot be drawn. Second, we do not have data regarding fetal cardiovascular profile of those participants. Our conclusions are based on the assumption that the observed changes in cardiac morphology and function began in utero as an adaptive response to chronic substrate deprivation. However, the findings of several studies on the cardiovascular profile of IUGR fetuses (4-7) support the validity of the above hypothesis. Third, LVCO determinations were performed by spectral Doppler, a technique that has the disadvantage of being operator dependent $(9,11)$. However, the method has been proven reliable in infants for the assessment of cardiac output in comparison with other invasive techniques (29). Moreover, any bias in LVCO measurements among our study subjects would be random. Additionally, the reliability of ejection fraction estimates depends largely on the accuracy of geometric assumptions regarding LV shape. Our results suggest that neonates exposed to IUGR present LV dilatation in a higher degree as compared with their AGA peers, and this difference in ventricular shape could theoretically affect ejection fraction estimates and comparisons. However, no differences in ejection fraction between AGA and IUGR infants were observed in this study, and this finding is in agreement with previous reports from neonates and children exposed to IUGR $(8,17,18)$. Finally, we utilized only the pulsed Doppler method to obtain TDI measurements, an approach that may have specific theoretical (10) and practical limitations when applied in neonates (11-13). Novel color-TDI modalities, such as isovolumic acceleration and strain-rate measurements, and 2D speckle tracking echocardiography, which are increasingly applied in adults (10), may also prove to be useful in children and infants in the future.

In conclusion, our study demonstrates that neonates exposed to IUGR present distinct changes in cardiac morphology and subclinical myocardial dysfunction, which results in an altered pattern of cardiovascular adaptation after birth. These findings further support the hypothesis that chronic intrauterine substrate deprivation is associated with a maladaptive programming process with persistent negative effects on the cardiovascular system. The potential clinical and prognostic implications of our findings remain to be established by future prospective studies with longer follow-up of these children. 


\section{METHODS}

\section{Study Design and Population}

This case-control study was conducted between January 2009 and July 2011 at the University General Hospital of Patras, Greece, a tertiary care referral institution that provides advanced Obstetric and Neonatal services in a region of $\sim 740,000$ inhabitants. The study population consisted of 30 neonates with IUGR and 30 controls cared for in the special care unit and well-baby nursery of our institution. Case subjects were otherwise healthy neonates with birth weight below the 10 th centile for gestational age who suffered IUGR documented by fetal ultrasound biometry (estimated fetal weight or abdominal circumference) and umbilical artery Doppler (30). The control group consisted of healthy neonates with birth weight AGA (i.e., birth weight between the 10th and 90th centile for gestational age) who were matched one-to-one with the IUGR infants according to gestational age. Gestational age was determined from the date of the last menstrual period in combination with early second-trimester ultrasound. Exclusion criteria for both groups were multiple gestation, congenital malformations or chromosomal aberrations, evidence of congenital or neonatal infection, congenital heart disease (including atrial septal defects), clinical or echocardiographical signs of pulmonary hypertension, perinatal asphyxia, tachypnea ( $>50$ breaths $/ \mathrm{min}$ ) or respiratory support requirement (other than oxygen supply for less than $12 \mathrm{~h}$ ), and need for volume expansion or administration of inotropes. Infants with a patent ductus arteriosus of a diameter $\geq 1.4 \mathrm{~mm} /$ $\mathrm{kg}$ or/and with left atrial-to-aortic root diameter ratio $\geq 1.4$ were considered as having hemodynamically significant patent ductus arteriosus (31) and were also excluded. The study was approved by the Ethics Committee of the University Hospital of Patras, and written parental consent was obtained for all participants prior to enrollment.

\section{Echocardiographic Studies}

Cardiac morphometry and function were assessed on the second and fifth postnatal day (24-36 and 96-120h, respectively) by the same pediatric cardiologist experienced in neonatal echocardiography (A.A.K.), using a Vivid 3 Pro system (GE Medical Systems, Milwaukee, WI) interfaced with a multifrequency probe (3.1-8.0 $\mathrm{MHz}$ ) with TDI function. Echocardiographic studies were performed with the neonates unsedated during quiet natural sleep after regular feeding. Electrocardiographic traces were recorded simultaneously to echocardiographic studies (built-in electrocardiographic recording function of the device) and used to determine heart rate at the time of the measurement. Images were stored in the hard drive of the device and were analyzed off-line by a second investigator (S.F.) who was blind to the clinical status of the neonates. Measurements were obtained from five consecutive cardiac cycles and averaged.

Conventional echocardiography was performed according to published standards (9). M-mode images were obtained from a parasternal long-axis view at the junction of the mitral valve leaflets and papillary muscles. IVS thickness at end diastole (IVSd), LV internal diameter at end systole (LVIDs), and end diastole (LVIDd), LV posterior wall thickness at end diastole (LVPWd), left atrial diameter at end systole, and internal diameter of the aortic annulus at end diastole were determined. Relative wall thickness was calculated as (IVSd + LVPWd)/LVIDd, fractional shortening as (LVIDd - LVIDs)/LVIDd $\times 100$, whereas ejection fraction estimates were automatically generated by the device (Teichholz formula). Mitral and tricuspid peak early diastolic (E) and late diastolic (A) flow velocities were determined. LVSV and LVCO were calculated using Doppler measurements obtained from the apical five-chamber view, with special care to minimize the angle between transducer beam and blood flow. The velocity-time integral (VTI) in the ascending aorta was determined, and LVSV and LVCO were calculated as aortic cross-sectional area $\times$ VTI and LVSV $\times$ heart rate, respectively. Ductus arteriosus patency and significance were assessed by a standardized approach (32).

Tissue Doppler imaging. Ventricular wall motion velocities were assessed by pulsed-wave TDI through an apical four-chamber view as previously described (11-13). Sample volumes were placed at the lateral margin of the mitral and tricuspid annulus with care to maintain an angle of $<20^{\circ}$. Peak systolic $\left(S^{\prime}\right)$, peak early diastolic ( $\left.E^{\prime}\right)$, and peak late diastolic (or atrial systolic; $\mathrm{A}^{\prime}$ ) myocardial velocities were determined. A typical example of a TDI recording is presented in Figure 1. Specific time intervals, such as isovolumetric contraction time, isovolumetric relaxation time, and ejection time were determined, and MPI (19) was calculated (Figure 1).

\section{Additional Investigations and Clinical Data}

Blood samples for complete blood count, chemistry, and BNP measurements (fluorescence immunoassay, Triage BNP test; BiositeDiagnositics, San Diego, CA) were obtained on the second and fifth postnatal day simultaneously with clinically indicated blood sampling. Blood pressure measurements were performed prior to echocardiographic examination with a Dinamap Critikon oscillometric device (Critikon, Tampa, FL) using cuffs of appropriate size. Three consecutive measurements were obtained and averaged.

Information regarding maternal demographics, pregnancy characteristics, and fetal ultrasound biometry were collected from obstetrical files. Data regarding neonatal characteristics and morbidities were also recorded. Clinical data are presented in detail in Table 1.

\section{Statistical Analysis}

Sample size estimations were based on preliminary measurements obtained from 10 IUGR-AGA pairs. These data revealed significant differences in MPI between the two groups, with the smaller difference noted in LV MPI (IUGR: 0.58 \pm 0.20 ; AGA: $0.42 \pm 0.18$; net difference: 0.16 ). Therefore, at least 30 IUGR-AGA pairs would be needed in order to detect a similar difference at a significance level of 0.05 with $90 \%$ power. Categorical variables were compared using the $\chi^{2}$ test (or the Fisher's exact test, as appropriate), whereas continuous variables were compared with the Mann-Whitney $U$-test. Comparisons between IUGR and AGA neonates on the same postnatal day were performed using multiple regression models with adjustment for potential confounding factors (gender, gestational age, birth weight, heart rate, smoking in pregnancy, gestational diabetes, hypertensive disorders of pregnancy, absent or reversed end-diastolic flow in umbilical artery Doppler, prenatal glucocorticoid administration, hemoglobin levels, and use of methylxanthines) (Supplementary Table S1 online). Between-group changes over time were assessed by two-way repeated measurements ANOVA, whereas within-group second-to-fifth postnatal day comparisons were performed by paired $t$-test. Intraobserver reliability was determined by calculating intraclass correlation coefficients using multiple echocardiographic measurements from 20 randomly selected neonates. This analysis showed high intraclass coefficients of agreement (between 0.87 and 0.93 - data not shown) for all echocardiographic parameters. Statistical analyses were performed using the SPSS software version 17.0 (SPSS, Chicago, IL)

\section{SUPPLEMENTARY MATERIAL}

Supplementary material is linked to the online version of the paper at http://www.nature.com/pr

\section{ACKNOWLEDGMENTS}

The authors acknowledge with gratitude the support of the medical and nursing stuff from the Neonatology Department of the University Hospital of Patras in the implementation of this study. They also thank Roland Neumann for his valuable suggestions.

Disclosure: The authors have no conflicts of interest or financial relationships relevant to this article to disclose.

\section{REFERENCES}

1. Rosenberg A. The IUGR newborn. Semin Perinatol 2008;32:219-24.

2. Barker DJ, Osmond C, Golding J, Kuh D, Wadsworth ME. Growth in utero, blood pressure in childhood and adult life, and mortality from cardiovascular disease. BMJ 1989;298:564-7.

3. Ross MG, Beall MH. Adult sequelae of intrauterine growth restriction. Semin Perinatol 2008;32:213-8.

4. Crispi F, Hernandez-Andrade E, Pelsers MM, et al. Cardiac dysfunction and cell damage across clinical stages of severity in growth-restricted fetuses. Am J Obstet Gynecol 2008;199:254.e1-8.

5. Comas M, Crispi F, Cruz-Martinez R, Martinez JM, Figueras F, Gratacós E. Usefulness of myocardial tissue Doppler vs conventional echocardiography 


\section{Cardiac dysfunction in IUGR neonates}

in the evaluation of cardiac dysfunction in early-onset intrauterine growth restriction. Am J Obstet Gynecol 2010;203:45.e1-7.

6. Comas M, Crispi F, Cruz-Martinez R, Figueras F, Gratacos E. Tissue Doppler echocardiographic markers of cardiac dysfunction in small-for-gestational age fetuses. Am J Obstet Gynecol 2011;205:57.e1-6.

7. Verburg BO, Jaddoe VW, Wladimiroff JW, Hofman A, Witteman JC, Steegers EA. Fetal hemodynamic adaptive changes related to intrauterine growth: the Generation R Study. Circulation 2008;117:649-59.

8. Leipala JA, Boldt T, Turpeinen U, Vuolteenaho O, Fellman V. Cardiac hypertrophy and altered hemodynamic adaptation in growth-restricted preterm infants. Pediatr Res 2003;162:426-427.

9. Lai WW, Geva T, Shirali GS, et al.; Task Force of the Pediatric Council of the American Society of Echocardiography; Pediatric Council of the American Society of Echocardiography. Guidelines and standards for performance of a pediatric echocardiogram: a report from the Task Force of the Pediatric Council of the American Society of Echocardiography. J Am Soc Echocardiogr 2006;19:1413-30.

10. Abraham TP, Dimaano VL, Liang HY. Role of tissue Doppler and strain echocardiography in current clinical practice. Circulation 2007;116:2597-609.

11. Ciccone MM, Scicchitano P, Zito A, et al. Different functional cardiac characteristics observed in term/preterm neonates by echocardiography and tissue doppler imaging. Early Hum Dev 2011;87:555-8.

12. Negrine RJ, Chikermane A, Wright JG, Ewer AK. Assessment of myocardial function in neonates using tissue Doppler imaging. Arch Dis Child Fetal Neonatal Ed 2012;97:F304-6.

13. Ekici F, Atalay S, Ozcelik N, Uçar T, Yilmaz E, Tutar E. Myocardial tissue velocities in neonates. Echocardiography 2007;24:61-7.

14. Matter M, Abdel-Hady H, Attia G, Hafez M, Seliem W, Al-Arman M. Myocardial performance in asphyxiated full-term infants assessed by Doppler tissue imaging. Pediatr Cardiol 2010;31:634-42.

15. Abdel-Hady HE, Matter MK, El-Arman MM. Myocardial dysfunction in neonatal sepsis: a tissue Doppler imaging study. Pediatr Crit Care Med 2012;13:318-23.

16. van der Hulst AE, Delgado V, Ten Harkel AD, et al. Tissue Doppler imaging in the left ventricle and right ventricle in healthy children: normal agerelated peak systolic velocities, timings, and time differences. Eur J Echocardiogr 2011;12:953-60.

17. Crispi F, Bijnens B, Figueras F, et al. Fetal growth restriction results in remodeled and less efficient hearts in children. Circulation 2010;121:242736.

18. Crispi F, Figueras F, Cruz-Lemini M, Bartrons J, Bijnens B, Gratacos E. Cardiovascular programming in children born small for gestational age and relationship with prenatal signs of severity. Am J Obstet Gynecol 2012;207:121.e1-9.

19. Tei C. New non-invasive index for combined systolic and diastolic ventricular function. J Cardiol 1995;26:19-23.

20. Tintu A, Rouwet E, Verlohren S, et al. Hypoxia induces dilated cardiomyopathy in the chick embryo: mechanism, intervention, and long-term consequences. PLoS ONE 2009;4:e5155.

21. Kiserud T, Ebbing C, Kessler J, Rasmussen S. Fetal cardiac output, distribution to the placenta and impact of placental compromise. Ultrasound Obstet Gynecol 2006;28:126-36.

22. Rouwet EV, Tintu AN, Schellings MW, et al. Hypoxia induces aortic hypertrophic growth, left ventricular dysfunction, and sympathetic hyperinnervation of peripheral arteries in the chick embryo. Circulation 2002;105:2791-6.

23. Tulloch NL, Muskheli V, Razumova MV, et al. Growth of engineered human myocardium with mechanical loading and vascular coculture. Circ Res 2011;109:47-59.

24. Wang KC, Zhang L, McMillen IC, et al. Fetal growth restriction and the programming of heart growth and cardiac insulin-like growth factor 2 expression in the lamb. J Physiol (Lond) 2011;589(Pt 19):4709-22.

25. Robel-Tillig E, Knüpfer M, Vogtmann C. Cardiac adaptation in small for gestational age neonates after prenatal hemodynamic disturbances. Early Hum Dev 2003;72:123-9.

26. Yasue $\mathrm{H}$, Yoshimura $\mathrm{M}$, Sumida $\mathrm{H}$, et al. Localization and mechanism of secretion of B-type natriuretic peptide in comparison with those of A-type natriuretic peptide in normal subjects and patients with heart failure. Circulation 1994;90:195-203.

27. El-Khuffash A, Molloy EJ. Are B-type natriuretic peptide (BNP) and N-terminal-pro-BNP useful in neonates? Arch Dis Child Fetal Neonatal Ed 2007;92:F320-4.

28. Koch A, Singer H. Normal values of B type natriuretic peptide in infants, children, and adolescents. Heart 2003;89:875-8.

29. Mellander M, Sabel KG, Caidahl K, Solymar L, Eriksson B. Doppler determination of cardiac output in infants and children: comparison with simultaneous thermodilution. Pediatr Cardiol 1987;8:241-6.

30. Figueras F, Gardosi J. Intrauterine growth restriction: new concepts in antenatal surveillance, diagnosis, and management. Am J Obstet Gynecol 2011;204:288-300.

31. Hamrick SE, Hansmann G. Patent ductus arteriosus of the preterm infant. Pediatrics 2010;125:1020-30.

32. Evans N. Diagnosis of the preterm patent ductus arteriosus: clinical signs, biomarkers, or ultrasound? Semin Perinatol 2012;36:114-22. 